Revue internationale P.M.E.

Économie et gestion de la petite et moyenne entreprise

\title{
La vision stratégique du propriétaire-dirigeant de PME: étude de cartographie cognitive
}

\section{Pierre Cossette}

Volume 9, numéro 1, 1996

URI : https://id.erudit.org/iderudit/1008257ar

DOI : https://doi.org/10.7202/1008257ar

Aller au sommaire du numéro

Éditeur(s)

Presses de l'Université du Québec

ISSN

0776-5436 (imprimé)

1918-9699 (numérique)

Découvrir la revue

Citer cet article

Cossette, P. (1996). La vision stratégique du propriétaire-dirigeant de PME: étude de cartographie cognitive. Revue internationale P.M.E., 9(1), 123-142. https://doi.org/10.7202/1008257ar
Résumé de l'article

Le concept de vision stratégique suscite depuis quelques années un intérêt grandissant, notamment dans les études portant sur la PME. Cependant, les techniques à la disposition des chercheurs et des consultants pour étudier empiriquement la vision stratégique des dirigeants d'entreprise sont encore très peu nombreuses. À cet égard, l'utilisation de la cartographie cognitive s'avère intéressante étant donné qu'elle facilite l'exploration des idées énoncées et qu'elle en rend possible une analyse particulière à l'aide, notamment, du logiciel COPE. C'est ce que cette recherche mettra en évidence à partir du cas d'un propriétaire-dirigeant d'une PME manufacturière québécoise. Certaines difficultés relatives à l'utilisation de la cartographie cognitive seront soulevées et quelques avenues de recherche seront envisagées. 


\section{La vision stratégique du propriétaire-dirigeant de PME: étude de cartographie cognitive}

Pierre COSSETTE

Université du Québec à Montréal

MOTS CLÉS

Carte cognitive - Cartographie cognitive-Vision stratégique PME - Propriétaire-dirigeant-Gestion stratégique-Cognition

\section{RÉSUMÉ}

Le concept de vision stratégique suscite depuis quelques années un intérêt grandissant, notamment dans les études portant sur la PME. Cependant, les techniques à la disposition des chercheurs et des consultants pour étudier empiriquement la vision stratégique des dirigeants d'entreprise sont encore très peu nombreuses. À cet égard, l'utilisation de la cartographie cognitive s'avère intéressante étant donné qu'elle facilite l'exploration des idées énoncées et qu'elle en rend possible une analyse particulière à l'aide, notamment, du logiciel COPE. C'est ce que cette recherche mettra en évidence à partir du cas d'un propriétaire-dirigeant d'une PME manufacturière québécoise. Certaines difficultés relatives à l'utilisation de la cartographie cognitive seront soulevées et quelques avenues de recherche seront envisagées.

\section{LES AUTEURS}

Pierre Cossette est professeur à l'École des sciences de la gestion de l'Université du Québec à Montréal. II est détenteur d'un Ph.D. en sciences de l'administration de l'Université Laval. II s'intéresse aux aspects cognitifs de l'organisation et en particulier à la cartographie cognitive comme outil de communication et d'analyse. Adresse : DSAUQAM, C.P. 6192, Succursale centre-ville, Montréal, (Québec), H3C 4R2. 


\begin{abstract}
The interest shown by researchers in the concept of strategic vision has grown in recent years, particularly in the area of small and medium-sized business. However, there are very few techniques available to researchers and consultants for empirical studies of the strategic vision of enterprise leaders. The use of cognitive mapping can be interesting because it facilitates the exploration of ideas expressed and makes a particular type of analysis possible, specially with the help of the software COPE. This will be highlighted in this research carried on with an owner-manager of a small manufacturing business in Québec. Difficulties related to the use of cognitive mapping will be raised and a number of avenues for further research will be suggested.
\end{abstract}

\title{
RESUMEN
}

El concepto de visión estratégica ha sido el objeto de un interés creciente los últimos años, particularmente en los estudios sobre la PyME. Sin embargo, es muy pequeña todavía la cantidad de técnicas a disposición de los investigadores y consultores para el estudio de la visión estratégica de los dirigentes de empresa. Al respecto, la utilización de la "cartografía cognoscitiva " puede ser interesante dado que ella facilita la exploración de ideas expresadas y ella hace posible un tipo de análisis particular, especialmente con la ayuda del programa COPE. Esto es lo que será destacado en esta investigación realizada a partir del caso de un dirigente de una PyME manufacturera quebequence. Se plantearán ciertas dificultades relativas a la utilización de la "cartografía cognoscitiva " y algunas perspectivas de investigación futura serán sugeridas. 


\section{Introduction}

Le concept de vision stratégique suscite depuis quelques années un intérêt grandissant et occupe maintenant une place de choix dans le vocabulaire de ce champ de connaissances qu'est la gestion stratégique. Bien qu'ambigu, ce concept a été au cœur d'un certain nombre d'études, le plus souvent à caractère conceptuel, qui ont à peu près exclusivement porté sur la grande entreprise.

Ces études, quoique intéressantes, mettent en évidence le peu de techniques à la disposition des chercheurs et des consultants pour étudier empiriquement la vision stratégique des dirigeants d'entreprise. Jusqu'à présent, les techniques employées ont semblé se limiter à l'entrevue en profondeur, à partir de questions plus ou moins dirigées ${ }^{1}$, et à la consultation de documents écrits ${ }^{2}$. À cet égard, l'utilisation de la cartographie cognitive pourrait s'avérer très intéressante étant donné qu'elle facilite l'exploration des idées énoncées et qu'elle en rend possible une analyse particulière ${ }^{3}$, notamment à l'aide du logiciel COPE développé par Colin Eden et son équipe et dont il sera question plus loin. Avant d'aborder les principaux éléments du cadre opératoire, je vais d'abord faire état des rares travaux dans le domaine des PME à l'intérieur desquels le concept de vision stratégique occupe une place centrale. Je définirai ensuite brièvement ce qu'est une carte cognitive et présenterai quelques études importantes de cartographie cognitive réalisées en contexte stratégique.

\section{Vision stratégique et PME}

Peu d'études dans le domaine des PME ont porté sur la vision stratégique des dirigeants. Il faut d'abord noter le travail de Filion (1991) pour qui la vision est « [...] une image, projetée dans le futur, de la place qu'on veut voir occupée éventuellement par ses produits sur le marché, ainsi que l'image du type d'organisation dont on a besoin pour y parvenir» (p. 109-110). Trois catégories de visions sont distinguées : émergente (qui représente les idées et concepts de produits et de services qu'un chef de file a dans la tête); centrale (qui est généralement l'aboutissement d'une ou de plusieurs visions émergentes), cette catégorie ayant une composante externe (la place qu'on veut voir occupée sur le marché par son produit ou service) et une composante interne (le type d'organisation créée pour y arriver); et secondaire (qui s'exprime par des activités de gestion en marketing, finance, etc.).

1. Voir, notamment, Filion (1991) et Carrière (1991).

2. Voir, notamment, Westley et Mintzberg (1989), bien que cette étude ne porte pas sur la PME.

3. Voir, notamment, Cossette et Audet (1992) ; Eden, Ackermann et Cropper (1992) ; Bougon, Weick et Binkhorst (1977) et Axelrod (1976). 
Il faut également souligner le travail de Carrière (1991) qui définit la vision stratégique comme «[...] la dynamique de construction mentale d'un futur souhaité et possible pour une entreprise» (p. 36). L'auteur précise trois niveaux différents dans la notion de vision : générale (valeurs et croyances du décideur), intermédiaire (schémas mentaux stratégiques) et parcellaire (aspects de l'environnement qui intéressent le décideur).

Enfin, pour d'Amboise et Bouchard (1990), la vision stratégique n'est pas le résultat d'un exercice de prédiction du futur. Elle est le fruit de la perspicacité du dirigeant. Elle renvoie essentiellement à une idée centrale et réaliste vers laquelle il veut mener son entreprise. Cette conception est en accord avec celle de Bennis et Nanus (1985) pour qui la vision est «[...] une image mentale d'un état futur possible et souhaitable de l'organisation» (p. 78).

Le concept semble porteur d'une richesse certaine, mais l'usage qu'on en fait, tant dans les études sur la PME que dans celles portant sur la grande entreprise, soulève au moins deux difficultés. D'abord, bien que la notion de vision stratégique désigne généralement un état futur à atteindre pour l'organisation, elle n'inclut pas toujours les facteurs susceptibles d'avoir une influence sur l'atteinte de cet état souhaité. Cette ambiguité demande à être levée étant donné qu'un concept peut difficilement faire référence à la fois à un ensemble (un but à atteindre et les moyens d'y parvenir) et à une partie de cet ensemble (uniquement le but à atteindre). Dans le cadre de cette recherche, les deux parties de l'ensemble seront prises en considération. De plus, il est habituellement présumé dans ces travaux que les dirigeants d'entreprise n'ont finalement qu'un seul but auquel ils associent un certain nombre de facteurs considérés par eux uniquement comme des moyens, ce qui pourrait être représenté de la façon suivante:

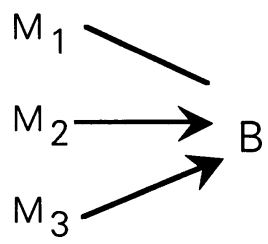

Pourtant, plusieurs études ${ }^{4}$ laissent croire que le mode de pensée des dirigeants d'entreprise est beaucoup plus complexe. Un même facteur peut très souvent être considéré à la fois comme un moyen et comme un but, étant donné qu'il exerce une influence sur d'autres et qu'il est lui-même influencé

4. Voir, notamment, Weick (1979) ; Huff (1990) ; Eden (1988) ; Weick et Bougon (1986) et Cossette et Audet (1992). 
par d'autres (ce que désigne bien l'expression anglaise means-ends chain). Plus encore, un facteur exerce parfois une influence sur lui-même par l'intermédiaire de un ou plusieurs autres facteurs, ce qui donne à la pensée de l'individu un caractère systémique ou circulaire. Schématiquement, on obtient alors ceci :

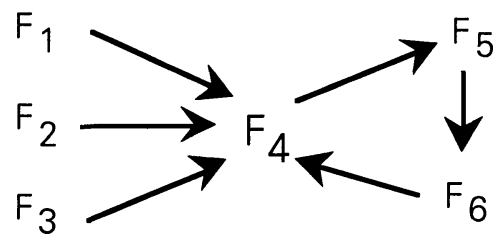

Dans la présente recherche, il ne sera pas présumé que la vision stratégique ne contient qu'un seul but, ni que certains facteurs sont uniquement des moyens et certains autres uniquement des buts, ni que les facteurs sont reliés entre eux de façon linéaire.

\section{Carte cognitive et gestion stratégique}

Une carte cognitive est une «représentation graphique de la représentation mentale que le chercheur se fait d'un ensemble de représentations discursives énoncées par un sujet à partir de ses propres représentations cognitives à propos d'un objet particulier» (Cossette et Audet, 1994, p. 15). Elle est une image constituée de concepts et de liens-le plus souvent d'influence-unissant certains d'entre eux, formant ainsi des sentiers d'influence, c'est-à-dire des chemins reliant un concept à un autre en passant par l'intermédiaire de un ou plusieurs autres concepts, ou même des boucles lorsqu'un concept exerce de façon indirecte une influence sur lui-même.

L'utilisation d'un tel outil suppose, le plus souvent, l'adoption d'une perspective interprétative ou subjectiviste. Cette perspective met l'accent sur les représentations que les individus se donnent de leur réalité plutôt que sur la réalité «objective», la réalité telle qu'elle serait vraiment. C'est le système référentiel de l'individu qui devient le centre d'intérêt. Dans cette optique, le chercheur va éviter d'imposer un cadre de référence a priori, c'est-à-dire de soumettre les sujets à des tests ou à des questionnaires, épreuves dans lesquelles les réponses sont classifiées dans des catégories prédéterminées. Il va plutôt chercher à rendre explicite la construction subjective que l'individu réalise de sa propre réalité, en présumant que c'est à partir d'elle qu'il interprète les événements, qu'il prend des décisions et qu'il agit.

L'utilisation de la cartographie cognitive dans le champ de la gestion stratégique est récente et a fait l'objet de peu d'études empiriques. Les travaux 
de Colin Eden et de ses collaborateurs ${ }^{5}$ constituent une exception notable. Pour eux, une carte cognitive est une représentation d'un modèle que le stratège possède de sa propre réalité, et la cartographie cognitive est un outil destiné fondamentalement à favoriser le processus de prise de décision stratégique en groupe. La carte cognitive (individuelle) ou la carte stratégique (collective) mettent principalement en évidence différentes options et différents objectifs stratégiques que se donnent des gestionnaires. D'autres recherches empiriques, dont celles de Hall (1984), de Stubbart et Ramaprasad (1988), de Diffenbach (1982), de Fiol et Huff (1992), de Barr, Stimpert et Huff (1992), de Calori, Johnson et Sarnin (1992) et de Bougon et Komocar (1994 a et b), ont montré que la cartographie cognitive pouvait permettre de mieux comprendre diverses situations stratégiques.

Toutefois, la cartographie cognitive a généralement été utilisée dans ces recherches dans le but d'aider des gestionnaires à définir leur situation stratégique actuelle ou à résoudre des problèmes importants auxquels ils faisaient face. Elle n'a pas été mise de façon systématique au service de l'étude de la vision stratégique des acteurs concernés. Pourtant, il y a là une avenue intéressante bien reconnue par Huff (1990, p. 41):

Cognitive mapping is particularly appropriate for exploring the vision in the mind of the CEO and other important figures [...] Mapping may help specify the content and process of strategy changes over time and help predict the direction of strategic action in the future.

C'est dans cette direction que s'oriente la présente recherche. Elle porte sur l'utilisation de la cartographie cognitive comme outil d'aide à la mise au point et à l'analyse de la vision stratégique chez un propriétaire-dirigeant de PME.

\section{Stratégie de recherche}

Avant d'aller plus loin, il est important de préciser la définition du concept de vision stratégique qui sera retenue ici :

La vision stratégique est un produit cognitif constitué d'un réseau de concepts jugés importants pour l'avenir de l'entreprise. Elle met en évidence un schème composé d' «explications» (causes ou moyens) et de «conséquences» (effets ou fins) qui guident les individus concernés dans l'interprétation des événements et dans les actions à entreprendre.

5. Voir, notamment, Eden, Jones, Sims et Gunton (1979) et Eden, Jones, Sims et Smithin (1981). 
Étant donné l'objet de la recherche et son caractère exploratoire, un seul propriétaire-dirigeant d'une PME manufacturière québécoise fut rencontré6. Au cours d'une première entrevue, il a été invité essentiellement à répondre aux trois questions suivantes:

1. «Qu'est-ce qui vous fera dire dans quelques années que votre entreprise a du succès ou non? En d'autres mots, quels sont les indices ou les manifestations qui permettront de dire dans quelques années que votre entreprise a du succès ou non?»

2. (Pour chacun des facteurs mentionnés en 1). «De quoi cela va-t-il dépendre si vous y arrivez ou non? En d'autres mots, quels sont les facteurs qui auront de l'influence sur l'atteinte ou non de ce succès? »

3. (Pour chacun des facteurs mentionnés en 1). «Sur quoi l'atteinte ou non de ce succès aura-t-elle une influence? Pourquoi est-ce important?»

Tout au long de l'entrevue enregistrée et ayant une durée d'un peu moins de 90 minutes, le propriétaire-dirigeant pouvait voir le chercheur tracer une première version de la carte cognitive et s'en servir comme outil d'exploration de ses propres idées.

Au cours de la deuxième entrevue, le propriétaire-dirigeant devait en quelque sorte «valider» la carte cognitive que lui présentait le chercheur. Ce dernier s'assurait que chacun des liens unissant différents concepts correspondait bien à ce que disait penser le propriétaire-dirigeant. Des ajouts ou autres modifications étaient apportés au besoin. Finalement, le chercheur lui demandait de considérer les facteurs mentionnés en réponse aux questions 2 et 3 de la première entrevue et de déterminer les facteurs qui les influencent ainsi que ceux sur lesquels ils exercent une influence. Toute cette procédure devait permettre de créer un réseau plus ou moins dense d'explications et de conséquences.

\section{Résultats}

L'analyse d'une carte cognitive repose essentiellement sur sa topographie, c'est-à-dire sur l'étude de la structure formée par les liens existant entre les concepts. En effet, les concepts ne prennent leur signification que dans leurs rapports avec d'autres concepts (Weick et Bougon, 1986; Eden, Jones et Sims,

6. Nous remercions Martyne Malo, alors étudiante à la maîtrise en administration, pour l'excellent travail qu'elle a accompli dans la réalisation des entrevues et dans la fabrication d'une première ébauche de la carte cognitive. 
1983; Bougon, 1983 ; Bougon, Weick et Binkhorst, 1977). C'est l'étude de ce réseau qui donne un sens à la carte cognitive.

L'analyse de la carte cognitive présentée dans cette recherche et réalisée avec l'aide du logiciel COPE a porté sur les quatre aspects suivants : l'importance relative de chacun des concepts, le regroupement des concepts, les facteurs influençants et les facteurs influencés et, enfin, les boucles. Elle est précédée d'une courte présentation du propriétaire-dirigeant et de son entreprise, à propos desquels certaines informations ont été modifiées pour respecter la confidentialité.

\section{Monsieur Lapointe...}

Monsieur Lapointe, 35 ans, est propriétaire-dirigeant d'une entreprise spécialisée dans la fabrication et l'aménagement de kiosques d'exposition. N'ayant pas de diplôme universitaire, monsieur Lapointe a appris «sur le tas » dans l'entreprise qu'il dirige depuis 1983. Fondée il y a 45 ans, l'entreprise emploie aujourd'hui 40 employés dont 30 sont directement affectés à la production. Tout en dirigeant l'ensemble de l'organisation, monsieur Lapointe est plus directement responsable des ventes et du marketing.

\subsection{Importance relative des concepts}

La littérature en cartographie cognitive suggère d'évaluer l'importance relative de chaque concept dans un graphique comme celui de la figure 1 à partir du nombre de facteurs auxquels un même concept est relié, directement ou indirectement, soit comme facteur influençant, soit comme facteur influencé. Un concept est donc considéré particulièrement important lorsqu'il possède beaucoup de liens avec d'autres, ce que Nozicka, Bonham et Shapiro (1976) désignent par l'expression cognitive centrality. Ainsi, Weick (1979) dira en faisant allusion aux liens directs entre les concepts dans une carte cognitive : «Plus un élément a d'intrants et/ou d'extrants, plus il est important» (p. 75). Dans Bougon et al. (1977), il est implicite que l'importance d'un concept peut être évaluée par l'utilisation d'une «matrice de proximité » (adjacency matrix) qui tient compte du nombre de facteurs qui lui sont directement reliés, ou par l'utilisation d'une «matrice de portée " (reachability matrix) où les liens indirects sont aussi pris en considération. Par ailleurs, Eden et al. (1983) ont développé une mesure plus complexe de l'importance de chaque concept dans une carte cognitive en vertu de laquelle, en plus de tenir compte du nombre total de concepts faisant office de facteurs influençants ou influencés directement ou indirectement, on prend en considération la longueur moyenne de tous les sentiers reliant ce concept à d'autres. Dans la présente étude, la manière de procéder suggérée par Weick ainsi que celle proposée par Eden et ses 
collaborateurs ont été retenues de façon à bien mettre en valeur les caractéristiques du schème représenté, compte tenu de la possibilité que certaines d'entre elles ne soient pas révélées par l'une ou l'autre de ces méthodes.

La carte cognitive (figure 1) de monsieur Lapointe comprend 40 concepts et 54 liens. Afin de ne pas alourdir le graphique (et tous les tableaux qui vont suivre), les concepts, qui sont des variables ou des construits constitués d'une idée et de celle qui lui est opposée, seront présentés dans leur forme la plus simple (par exemple, «niveau des ventes » plutôt que «niveau plus ou moins élevé des ventes»). Quant aux liens, ils prennent la forme d'un trait se terminant par une pointe pleine $(\rightarrow)$ si la relation perçue est positive (par exemple, une hausse de $\mathrm{A}$ conduit à une hausse de $\mathrm{B}$ ) et par une pointe creuse $(\rightarrow)$ si cette relation est négative (par exemple, une hausse de $\mathrm{A}$ conduit à une baisse de B). L'importance de chacun des facteurs telle que reflétée par le nombre total de liens directs les unissant à d'autres facteurs a été déterminée. Les 11 concepts les plus importants sont présentés dans le tableau 1. Le tableau 2 montre les 11 concepts ayant obtenu le plus haut score d'après le nombre de liens, directs et indirects, et la longueur des sentiers qui les rattachent aux autres concepts.

D'entrée de jeu, il est facile de constater que monsieur Lapointe a des préoccupations variées relativement à sa vision stratégique. Ce n'est guère surprenant étant donné la centralisation de la prise de décision dans les PME (Welsh et White, 1981; MacMillan, 1975; d'Amboise et Muldowney, 1989).

On remarque également que l'importance de chaque concept varie relativement peu d'un tableau à l'autre. Étant donné qu'en procédant de la façon suggérée par Weick, l'importance de chaque concept est évaluée sur la base de la conscience discursive qu'à l'individu de chacun des liens de sa carte cognitive, ce qui n'est pas le cas avec la méthode proposée par Eden et al. (par exemple, l'individu peut ne pas avoir remarqué dans sa propre carte cognitive qu'un concept exerce un impact sur lui-même ou sur un autre par l'intermédiaire de 15 autres concepts), on doit reconnaître chez monsieur Lapointe une certaine cohérence sur le plan cognitif. La place plus ou moins centrale qu'occupe un concept dans sa carte cognitive varie très peu, qu'elle soit attribuée ou non à partir de la conscience explicite qu'il a des liens de sa carte cognitive.

Par ailleurs, les concepts les plus importants dans chacun des deux tableaux montrent chez monsieur Lapointe un intérêt marqué pour la croissance de son entreprise et laissent croire qu'elle passe avant tout par une qualité supérieure du produit et une forte implication du personnel. Parmi les six plus importants facteurs dans chacun des deux tableaux, cinq sont les mêmes, soit «développement de nouvelles clientèles », «niveau des ventes», «qualité des produits», «implication du personnel dans son travail» et «petite taille de 


\section{FIGURE 1}

\section{Carte cognitive de monsieur Lapointe}

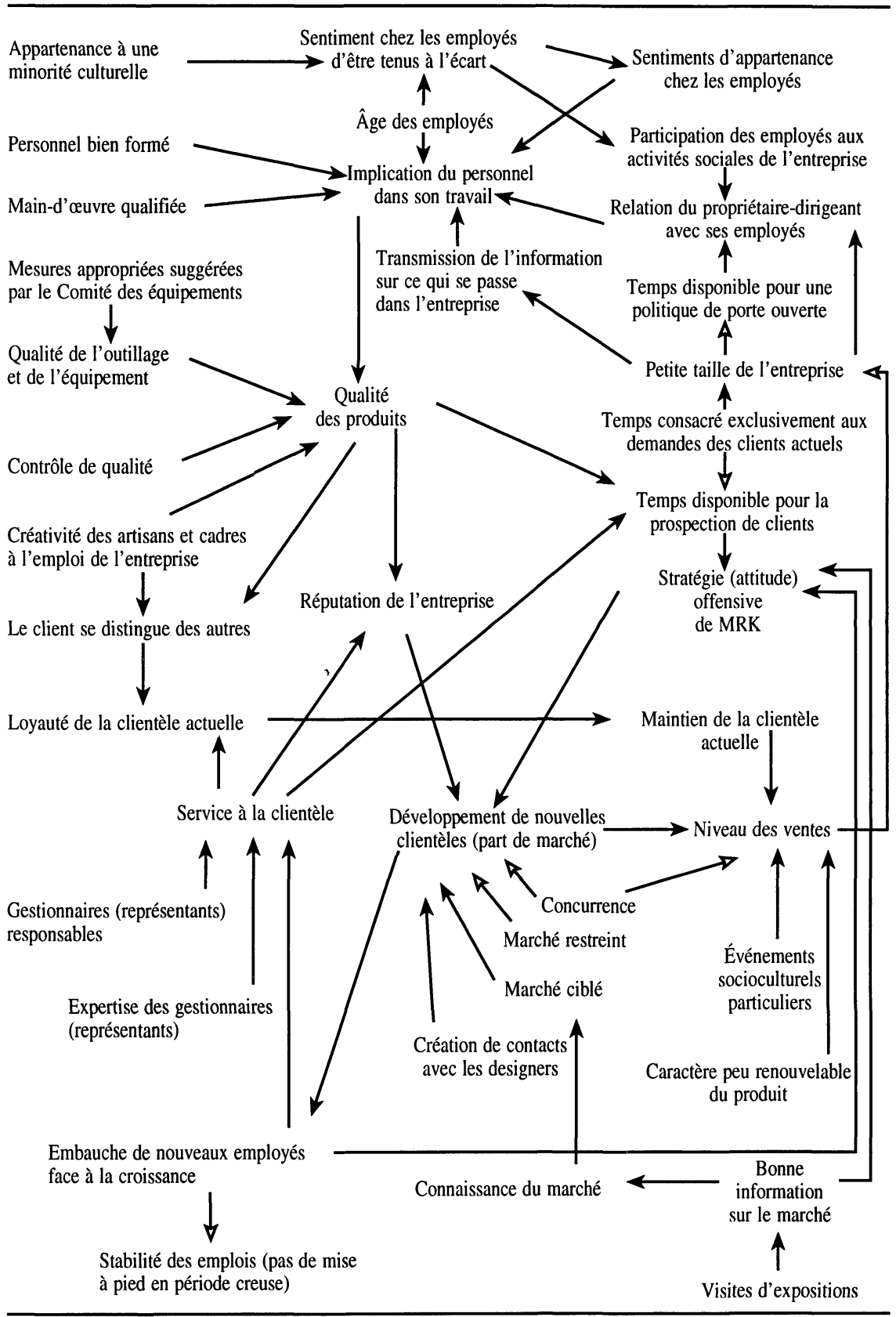


Tableau 1

Nombre total de liens directs unissant les 11 concepts les plus importants aux autres

\begin{tabular}{lr} 
Développement de nouvelles clientèles (part de marché) & 8,0 \\
Implication du personnel dans son travail & 7,0 \\
Qualité des produits & 7,0 \\
Service à la clientèle & 6,0 \\
Niveau des ventes & 6,0 \\
Petite taille de l'entreprise & 5,0 \\
Sentiment chez les employés d'être tenus à l'écart & 4,0 \\
Relation du propriétaire-dirigeant avec ses employés & 4,0 \\
Temps disponible pour la prospection de clients & 4,0 \\
Stratégie (attitude) offensive de marketing & 4,0 \\
Embauche de nouveaux employés face à la croissance & 4,0 \\
\hline
\end{tabular}

TABleau 2

Importance relative des 11 concepts les plus importants selon le nombre de liens et la longueur des sentiers qui les unissent aux autres

\begin{tabular}{lr}
\hline Niveau des ventes & 116,8 \\
Petite taille de l'entreprise & 112,2 \\
Développement de nouvelles clientèles (part de marché) & 112,1 \\
Qualité des produits & 108,9 \\
Implication du personnel dans son travail & 107,4 \\
Relation du propriétaire-dirigeant avec ses employés & 101,9 \\
Temps disponible pour la prospection de clients & 101,2 \\
Transmission de l'information sur ce qui se passe dans l'entreprise & 100,3 \\
Embauche de nouveaux employés face à la croissance & 100,0 \\
Loyauté de la clientèle actuelle & 99,3 \\
Temps consacré exclusivement aux demandes des clients actuels & 99,3 \\
\hline
\end{tabular}

l'entreprise». Le fait que le niveau des ventes et le développement de nouvelles clientèles soient des facteurs prépondérants dans sa carte cognitive révèle également son orientation vers les résultats, une caractéristique reconnue chez les propriétaires-dirigeants de PME (Gasse, 1982). De plus, la place centrale qu'occupent ces deux facteurs dans la carte cognitive de monsieur Lapointe laisse penser qu'il est peut-être plus motivé par le défi de la croissance que par le succès lui-même, une attitude déjà notée par DeCarlo et Lyons (1980) dans une étude sur les propriétaires-dirigeants de PME. 
Finalement, il faut noter que parmi les facteurs exerçant, selon monsieur Lapointe, une influence directe sur le développement de nouvelles clientèles ou sur le niveau des ventes, il y en a plusieurs qui semblent hors de son contrôle : la concurrence, le fait que le marché soit restreint, la présence d'événements socioculturels particuliers et le caractère peu renouvelable du produit. Cette constatation dénote la présence chez monsieur Lapointe d'une vision un peu fataliste de l'avenir de l'entreprise ou encore l'existence d'un sentiment plutôt moyen de pouvoir contrôler son destin (internal locus of control) alors que ce sentiment est généralement considéré comme élevé chez les propriétairesdirigeants de PME (Gasse, Brouard et Sy, 1985). Toutefois, la majorité des concepts de la carte cognitive semblent susceptibles d'être affectés par les actions de monsieur Lapointe, ce qui témoigne d'une vision stratégique plus volontariste que déterministe.

\subsection{Regroupement des variables}

L'étude des caractéristiques générales d'une carte cognitive est souvent enrichie par une analyse de regroupement (clustering analysis) sur la base de la similitude des liens entre les concepts (Eden, Jones et Sims, 1983). Cet outil statistique peut faire apparaître des dimensions particulières qui n'auraient pas été mises en valeur autrement.

Dans le cas présent, l'analyse de regroupement a fait ressortir trois groupes de concepts qui ne peuvent toutefois être présentés ici, faute d'espace. Les 10 concepts du groupe 1 renvoient principalement aux caractéristiques des employés (âge, implication, qualifications, etc.), alors que les 12 concepts du groupe 2 ont plutôt trait aux processus de gestion. La stratégie semble être le thème principal autour duquel gravitent les 18 variables du groupe 3.

En somme, le personnel, les processus de gestion et la stratégie constituent les trois grandes dimensions à partir desquelles monsieur Lapointe paraît envisager l'avenir de son entreprise. Il s'agit d'une caractéristique particulière de son schème d'interprétation et d'action tel qu'il est représenté par la carte cognitive.

\subsection{Facteurs influençants et facteurs influencés}

L'analyse a aussi porté sur les caractéristiques des facteurs influençants et des facteurs influencés. L'appartenance de certains concepts à l'une ou l'autre de ces catégories n'est pas l'effet du hasard. Ainsi, certains facteurs tendent à être fortement considérés comme des extrants, c'est-à-dire comme des facteurs sur lesquels plusieurs autres exercent directement une influence. En d'autres mots, ces concepts se présentent d'abord comme des conséquences ou des résultats 
plutôt que comme des explications. Par ailleurs, d'autres facteurs tendent à être considérés principalement comme des intrants, c'est-à-dire comme des facteurs exerçant une influence directe sur plusieurs autres facteurs. Les tableaux 3 et 4 montrent respectivement les facteurs influencés directement par au moins trois autres facteurs et les facteurs influençant directement au moins trois autres facteurs.

\section{TABlEAu 3}

Facteurs influencés directement par au moins trois autres facteurs

\begin{tabular}{lr}
\hline Développement de nouvelles clientèles & 6 \\
Implication du personnel dans son travail & 6 \\
Niveau des ventes & 5 \\
Qualité des produits & 4 \\
Service à la clientèle & 3 \\
Relation du propriétaire-dirigeant avec ses employés & 3 \\
Temps disponible pour la prospection de clients & 3 \\
Stratégie (attitude) offensive de marketing & 3 \\
\hline
\end{tabular}

\section{TABLEAU 4}

Facteurs influençant directement au moins trois autres facteurs

$\begin{array}{ll}\text { Petite taille de l'entreprise } & 4 \\ \text { Qualité des produits } & 3 \\ \text { Service à la clientèle } & 3 \\ \text { Embauche de nouveaux employés face à croissance } & 3\end{array}$

On remarque d'abord qu'il y a deux fois plus de concepts dans le tableau 3 que dans le tableau 4 . Il est possible que ce phénomène reflète un intérêt particulier pour les résultats, une tendance déjà notée précédemment. Par ailleurs, deux concepts se trouvent à la fois dans les tableau 3 et $4:$ qualité des produits et service à la clientèle. Ils peuvent être considérés comme des éléments clés de la vision stratégique de monsieur Lapointe. En étant des explications $e t$ des conséquences jugées importantes par le nombre de liens les unissant à d'autres, ils deviennent les plus susceptibles de faire partie de relations d'influence circulaires, conférant ainsi au système représenté un aspect dynamique.

Il faut signaler finalement que la petite taille de l'entreprise est considérée comme le facteur explicatif dominant dans la carte cognitive. Elle exercerait, selon monsieur Lapointe, une influence sur les quatre facteurs suivants: le temps disponible pour une politique de porte ouverte, le temps consacré exclusivement aux demandes des clients actuels, la relation de 
proximité du propriétaire-dirigeant avec ses employés et la transmission de l'information sur ce qui se passe dans l'entreprise.

\subsection{Boucles}

Au-delà des relations d'influence directes, les concepts et liens forment parfois des sentiers, c'est-à-dire des chemins reliant un concept à un autre en passant par l'intermédiaire de un ou plusieurs autres concepts. Un sentier va créer un cycle ou une boucle lorsque le concept final est relié au concept initial, c'est-àdire lorsqu'un facteur exerce, de façon indirecte, une influence sur lui-même. Une boucle est dite positive lorsqu'elle ne contient aucun lien négatif ou lorsqu'elle en contient un nombre pair, et elle est dite négative si ce nombre est impair. Ainsi, dans une boucle positive, il y a un effet d'amplification de la tendance initiale existant entre chaque paire de concepts. Ce mouvement en spirale exerce un effet déstabilisateur sur le système parce qu'il est monotone, c'est-à-dire que la valeur prise par chaque concept change toujours dans la même direction une fois le mouvement de départ déclenché. Dès lors, le système s'emballe et peut devenir non contrôlable. Suivant la même logique, une boucle négative a un effet stabilisateur sur le système (Maruyama, 1963), parce que la dynamique du système fait augmenter et diminuer-ou vice versa-alternativement la valeur de chaque concept.

La figure 2 fournit une représentation graphique des boucles présentes dans la carte cognitive de monsieur Lapointe. Elle contient les 17 concepts engagés dans la formation d'au moins une boucle. Le fait que seulement trois liens soient négatifs et que, par conséquent, le nombre de boucles positives soit vraisemblablement beaucoup plus élevé que celui des boucles négatives autorise à penser que le changement caractérise beaucoup plus cette entreprise que la stabilité. Cela serait en accord avec l'objectif de croissance poursuivi par monsieur Lapointe.

Par ailleurs, on ne s'étonne pas que ce soit surtout avec des facteurs associés à la vente et à la clientèle que monsieur Lapointe possède une pensée circulaire. En assumant lui-même la responsabilité du marketing dans l'entreprise, on comprend que ce soit avec ces facteurs qu'il entretient une logique plus complexe, un mode de pensée plus systémique.

Enfin, même si la figure contient les principaux concepts autour desquels semble s'organiser la vision stratégique de monsieur Lapointe, il est intéressant de noter que la plupart des facteurs exerçant une influence sur le développement de nouvelles clientèles et sur le niveau des ventes ne s'inscrivent pas dans un mode de pensée circulaire chez monsieur Lapointe. Il en est de même de la majorité des facteurs ayant un impact sur l'implication du personnel dans son travail. 
FIGURE 2

\section{Boucles présentes dans la carte cognitive de monsieur Lapointe}

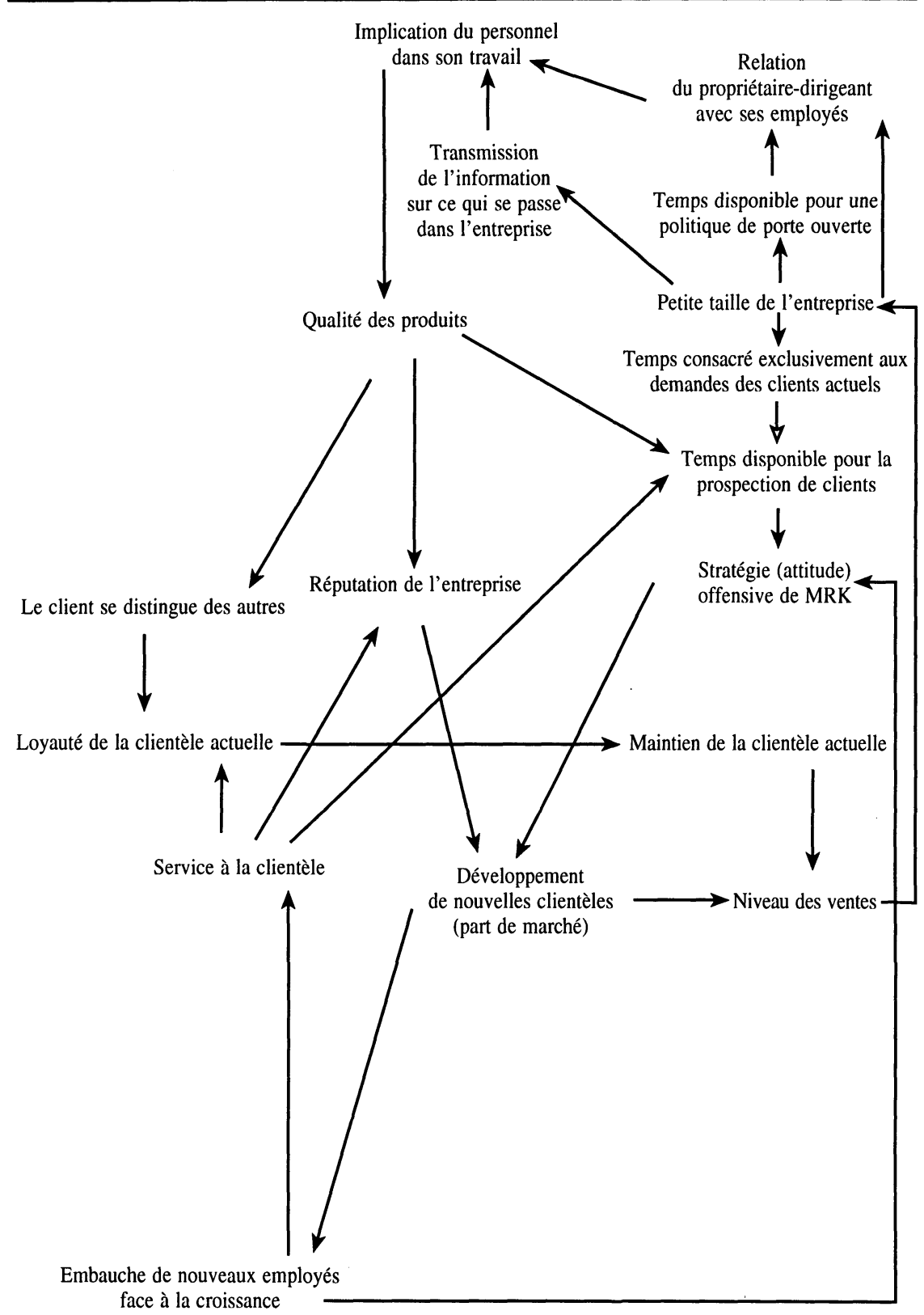

face à la croissance 


\section{Conclusion}

Cette recherche a montré que la cartographie cognitive pouvait aider le propriétaire-dirigeant de PME à mettre au point et à analyser sa vision stratégique. Cet outil permet notamment de mettre en évidence des caractéristiques qu'il aurait été difficile, voire impossible, de déterminer autrement. Compte tenu qu'il facilite l'élucidation de l'objet étudié et qu'il possède des propriétés émancipatoires (Audet, 1989; 1994), cet instrument est susceptible de conduire à des interventions plus éclairées sur le plan stratégique.

Cependant, étant donné que la présente recherche ne portait que sur un seul cas, la prudence s'impose. Il faudra étudier de nouveaux cas pour mieux cerner le caractère instrumental de la cartographie cognitive dans un tel contexte. Par exemple, il est possible que cet outil aide davantage le propriétaire-dirigeant possédant certaines caractéristiques comme une forte capacité d'introspection et une grande habileté à s'exprimer.

Par ailleurs, l'utilisation de la cartographie ne se fait pas sans difficultés et soulève de nombreuses questions, ce qui fait apparaitre de nouvelles pistes de recherche en ce qui a trait à l'outil lui-même. Ces difficultés et questions dont j'ai déjà fait état ailleurs (voir Cossette, 1989; Cossette et Audet, 1992) portent principalement sur les aspects suivants : l'utilisation exclusive d'un critère quantitatif pour évaluer l'importance d'un concept; l'intensité non mesurée de l'influence d'un concept sur un autre; l'absence de considération pour les relations contingentes (dans lesquelles un concept exerce un impact sur un lien plutôt que sur un autre concept, ou encore lorsqu'un lien plutôt qu'un concept exerce une influence sur un concept ou même sur un autre lien), interactionnelles (dans lesquelles la combinaison de différents facteurs produit une conséquence qu'aucun des concepts pris isolément n'aurait entraînée) et non monotones (dans lesquelles la relation entre deux concepts possède un caractère curviligne); et, finalement, la signification du lien d'influence. (L'influence estelle «actuelle» ou «possible»? L'explication est-elle une «cause» déclenchant un certain «effet» ou un «moyen» en vue d'une «fin» particulière?)

En plus de contribuer à d'éventuels développements sur le plan méthodologique, la recherche future devra se pencher sur le lien existant entre, d'une part, les idées représentées dans une carte cognitive et, d'autre part, les actions et interprétations des individus. De telles orientations permettront d'évaluer dans quelle mesure une carte cognitive peut aider à comprendre tant les actions qui seront posées que les interprétations qui seront fournies.

Ainsi, à propos du lien particulier entre la « carte cognitive» représentant la vision stratégique et les «actions stratégiques » posées, il convient de rappeler la distinction établie par Argyris et Schön (1974) entre «théorie adoptée» 
(espoused theory) et «théorie utilisée » (theory-in-use). Même en présumant de la volonté du sujet d'être sincère, il n'est pas impossible que certaines parties du schème qu'il dit posséder et qui est représenté par une carte cognitive diffèrent des idées qui le guident dans ses actions. Les conditions de la plus ou moins grande cohérence entre la carte cognitive et les décisions stratégiques ainsi que les caractéristiques du lien plus ou moins étroit entre les deux méritent d'être explorées.

Quant à la relation existant entre la carte cognitive et l'interprétation que l'individu fait des événements, les conditions et les caractéristiques de cette relation plus ou moins grande entre les deux ne sont guère connues. Les théories de l'attribution ont clairement mis en évidence la tendance qu'auraient les gens à attribuer les conséquences agréables à des facteurs internes ou personnels et les conséquences désagréables à des facteurs externes ou environnementaux (Kelley et Michela, 1980), mais elles n'ont pas établi dans quelle mesure cette tendance était congruente avec les représentations discursives passées des individus, surtout si la représentation graphique qui en a été faite a été endossée par eux.

Également, il serait intéressant de comparer différentes cartes cognitives représentant la vision stratégique de propriétaires-dirigeants de PME en fonction des caractéristiques personnelles de ces derniers. Ainsi, le style cognitif, les valeurs, les motifs pour se lancer et demeurer en affaires ainsi que certains traits de personnalité comme le sentiment plus ou moins fort de maîtriser son destin mettent en relief des particularités individuelles qui ne sont peut-être pas étrangères à la structure et au contenu des cartes cognitives. Il y a là des voies de recherche qui méritent d'être empruntées.

L'analyse d'une carte cognitive elle-même peut être enrichie. Il faudra mettre davantage en relation les différents résultats qu'on peut obtenir à l'aide du logiciel COPE. L'étude de tels liens permettrait notamment de faire ressortir la cohérence plus ou moins grande de ces différents résultats.

Enfin, la mise au point d'une procédure plus systématique qui permettrait à l'individu de tracer lui-même sa carte cognitive et de la faire parvenir éventuellement à un spécialiste chargé d'en faire l'analyse serait de nature à favoriser la réflexion stratégique chez les décideurs concernés. Ce serait là un pas important dans le développement d'outils et de services d'aide à la gestion stratégique. 


\section{Bibliographie}

ARGYRIS, C. et D.A. SCHÖN (1974), Theory in Practice: Increasing Professional Effectiveness, San Francisco, Jossey-Bass.

AUdET, M. (1989), «La cartographie cognitive et ses objets». Communication présentée au congrès de l'Association canadienne-française pour l'avancement des sciences (ACFAS), Montréal.

AudET, M. (1994), «Plasticité, instrumentalité et réflexivité», dans P. Cossette (dir.), Cartes cognitives et organisations, Québec, Les Presses de l'Université Laval, collection «Sciences de l'administration», Paris, Éditions ESKA, p. 187-198.

AXElRod, R. (dir.), (1976), Structure of Decision: The Cognitive Maps of Political Elites, Princeton, N. J., Princeton University Press.

BARR, P.M., J.L. STIMPERT et A.S. HufF (1992), « Cognitive change, strategic action, and organizational renewal », Strategic Management Journal, vol. 13, p. 15-36.

BenNis, W. et B. NANUS (1985), Diriger, Paris, InterÉditions.

Bougon, M.G., K.E. WeICK et D. BINKHORST (1977), « Cognition in organizations : an analysis of the Utrecht Jazz Orchestra », Administrative Science Quarterly, vol. 22, p. 606-639.

Bougon, M.G. (1983), «Uncovering cognitive maps: the Self-Q technique», dans G. Morgan (dir.), Beyond Method. Strategies for Social Research, Beverly Hills, Sage, p. 173-188.

Bougon, M.G. et J.M. Komocar (1994a), «Les cartes cognitives composites. Une théorie holistique et dynamique des organisations et du processus d'organisation », dans P. Cossette (dir.), Cartes cognitives et organisations, Québec, Les Presses de l'Université Laval, collection «Sciences de l'administration », Paris, Éditions ESKA, p. 37-56.

Bougon, M.G. et J.M. KomocaR (1994b), «Façonner et diriger la stratégie. Approche holistique et dynamique », dans P. Cossette (dir.), Cartes cognitives et organisations, collection «Sciences de l'administration», Québec, Les Presses de l'Université Laval, Paris, Éditions ESKA, p. 57-80.

CALORI, R., G. JOHNSON et P. SARNIN (1992), «French and British top managers' understanding of the structure and the dynamics of their industries : a cognitive analysis and comparison », British Journal of Management, vol. 3, p. 61-78.

CARRIÈre, J.B. (1991), «La vision stratégique en contexte de PME: cadre théorique et étude empirique», Revue Internationale PME, vol. 4, $\mathrm{n}^{\circ} 1$, p. 34-56.

Cossette, P. (1989), Les schèmes d'interprétation idiosyncratiques de propriétairesdirigeants de PME : une étude de cartographie cognitive, Thèse de doctorat inédite, Québec, Université Laval. 
Cossette, P. et M. Audet (1992), « Mapping of an idiosyncratic schema », Journal of Management Studies, vol. 20, n 3, p. 325-347.

Cossette, P. et M. Audet (1994), «Qu'est-ce qu'une carte cognitive? », dans P. Cossette (dir.), Cartes cognitives et organisations, Québec, Les Presses de l'Université Laval, collection «Sciences de l'administration », Paris, Éditions ESKA, p. 13-33.

D'Amboise, G. et M. Muldowney (1988), « Management theory for small business : attempts and requirements », Academy of Management Review, vol. 13, n² 2, p. 226-240.

D'Amboise, G. et S. Bouchard (1990), «De l'utilisation du concept de vision dans l'étude des organisations », Document de travail, Québec, Université Laval (FSA).

DeCARLO, J.F. et P.R. LYONS (1980), «Toward a contingency theory of entrepreneurship », Journal of Small Business Management, vol. 18, $\mathrm{n}^{\circ} 3$, p. 37-42.

DifFENBACH, J. (1982), «Influence diagrams for complex strategic issues », Strategic Management Journal, vol. 3, p. 133-146.

Eden, C., S. Jones, D. Sims et H. Gunton (1979), «Images into models: the subjective world of the policy maker $»$, Futures, vol. 11, nº 1, p. 56-62.

Eden, C., S. Jones, D. Sims et T. SMITHIN (1981), «The intersubjectivity of issues and issues of intersubjectivity », Journal of Management Studies, vol. 18, $\mathrm{n}^{\circ} 1$, p. 37-47.

Eden, C., S. Jones et D. Sims (1983), Messing About in Problems, Oxford, Pergamon.

EDEN, C. (1988), «Cognitive mapping : a review », European Journal of Operational Research, vol. 36, p. 1-13.

Eden, C., F. ACKermann et S. CROPPER (1992), "The analysis of cause maps », Journal of Management Studies, vol. 29, n 3, p. 309-324.

FILION, L.J. (1991), Vision et relations : clefs du succès de l'entrepreneur, Montréal, Les Éditions de l'Entrepreneur.

FIOL, C.M. et A.S. HufF (1992), «Maps for managers : Where are we ? Where do we go from there?», Journal of Management Studies, vol. 29, $\mathrm{n}^{\circ} 3$, p. 267-285.

GASSE, Y. (1982), «L'entrepreneur moderne : attributs et fonctions », Gestion, Revue Internationale de Gestion, vol. 7, $\mathrm{n}^{0} 4$, p. 3-10.

GASSE, Y., J.F. BROUARD et A. SY (1985), «L'entrepreneurship : une stratégie de recherche et d'intervention pour le développement ", Revue PMO, vol. 1, n ${ }^{0} 5$, p. 8-24.

HALL, R.I. (1984), «The natural logic of management policy making: its implications for the survival of an organisation », Management Science, vol. 30, p. 905-927. 
HuFf, A.S. (dir.), (1990), Mapping Strategic Thought, Chichester, Angleterre, John Wiley and Sons.

Kelley, H.H. et J.L. Michela (1980), «Attribution theory and research », Annual Review of Psychology, vol. 31, p. 457-501.

MACMILlaN, I.C. (1975), «Strategy and flexibility in the smaller business », Long Range Planning, vol. $8, \mathrm{n}^{\circ} 3$, p. 62-63.

MARUYAMA, M. (1963), "The second cybernetics: deviation-amplifying mutual cause processes », American Scientist, vol. 51, p. 164-179.

Nozicka, G., G.M. BonhaM et M.J. ShaPIRo (1976), « Simulation techniques », dans R. Axelrod (dir.), Structure of Decision: The Cognitive Maps of Political Elites, Princeton, N. J., Princeton University Press, p. 349-359.

STUBbarT, C.I. et A. RAMAPRASAD (1988), «Probing two chief executives schematic knowledge of the U.S. steel industry using cognitive maps », dans R. Lamb et P. Skirvastava (dir.), Advances in Strategic Management, Greenwich, Connecticut, JAI Press, p. 139-164.

WeICK, K.E. (1979), The Social Psychology of Organizing $2^{\mathrm{e}}$ édition, Reading, Massachusetts, Addison-Wesley.

WEICK, K.E. et M.G. BougON (1986), «Organizations as cognitive maps : charting ways to success and failure», dans H.P. Sims, Jr. et D.A. Gioia (dir.), The Thinking Organization: Dynamics of Organizational Social Cognition, San Francisco, Jossey-Bass, p. 102-135.

WeLSh, J.A. et J.F. White (1981), "A small business is not a little big business », Harvard Business Review, vol. 59, $\mathrm{n}^{\circ} 4$, p. 18-32.

WeStley, F. et H. MinTzBERG (1989), «Visionary leadership and strategic management », Strategic Management Journal, vol. 10, p. 17-32. 